\title{
VARIACIÓN DE RASGOS DEL XILEMA SECUNDARIO DE BULNESIA RETAMA (ZYGOPHYLLACEAE) A LO LARGO DE UN GRADIENTE DE ARIDEZ EN EL CENTRO-OESTE DE ARGENTINA
}

\author{
Edgardo A. Melián ${ }^{1,2}$ (D), Gabriel Gatica ${ }^{1,3}$ (iD) \& Eduardo Pucheta ${ }^{*}$ (iD
}

\begin{abstract}
${ }^{1}$ Departamento de Biología, Facultad de Ciencias Exactas, Físicas y Naturales, Universidad Nacional de San Juan, Av. Ignacio de la Roza 590 (O), J5402DCS Rivadavia, San Juan, Argentina; *epucheta@unsj-cuim.edu.ar (autor corresponsal). ${ }^{2}$ Becario doctoral del CONICET.

${ }^{3}$ Centro de Investigaciones en Física e Ingeniería del Centro de la Provincia de Buenos Aires, CONICET, Comisión de Investigaciones Cientificas de la provincia de Buenos Aires, Universidad Nacional del Centro de la Provincia de Buenos Aires, Pinto 399, B7000GHG Tandil, Buenos Aires, Argentina.
\end{abstract}

Abstract. Melián, E. A.; G. Gatica \& E. Pucheta. 2021. Variation in Bulnesia retama (Zygophyllaceae) secondary xylem traits along an aridity gradient in central-western Argentina. Darwiniana, nueva serie 9(1): 147-161.

The study of secondary xylem of Bulnesia retama along an aridity gradient allowed finding variations among different xylem cell types that can be used to predict tolerance of this species to aridity. The aim of this work was to carry out an anatomical description of the secondary xylem through cross sections, to analyse the variation of traits considered to be important for survival in arid environments, such as the percentage of vessels, fibres and parenchyma, fibre wall thickness, and wood density. The samples were obtained from primary branches of plants from four locations along an aridity gradient (Médanos Grandes, Bermejo, Marayes and Chepes, San Juan and La Rioja provinces, Argentina). Cross sections were observed with optical microscope and resulting digital images were analysed with ImageJ software. The results indicate that vessels are small, numerous and diagonally distributed, axial parenchyma is apotracheal diffuse. A significant increase in total fibre area and a decrease in parenchyma area with increases in aridity were observed. Total vessel area did not show any changes, while fibre wall thickness increased slightly with aridity. These traits suggest that $B$. retama has a highly drought-tolerant wood showing phenotypic plasticity along the aridity gradient, also registering a high-density xeromorphic wood.

Keywords. Arid lands; cross-section area; environmental gradient; xylematic traits.

Resumen. Melián, E. A.; G. Gatica \& E. Pucheta. 2021. Variación de rasgos del xilema secundario de Bulnesia retama (Zygophyllaceae) a lo largo de un gradiente de aridez en el centro-oeste de Argentina. Darwiniana, nueva serie 9(1): 147-161.

El estudio del xilema secundario de Bulnesia retama a lo largo de un gradiente de aridez permite encontrar variaciones entre los diferentes tipos celulares del xilema que predicen la tolerancia de esta especie a la aridez. El objetivo de este trabajo fue realizar una descripción anatómica del xilema secundario mediante cortes transversales, analizar la variación de rasgos considerados de importancia para la supervivencia en ambientes áridos, como el porcentaje de vasos, fibras y parénquima y el grosor de la pared de fibras, y también la densidad del leño. Las muestras se obtuvieron de las ramas primarias de plantas provenientes de cuatro localidades a lo largo de un gradiente de aridez (Médanos Grandes, Bermejo, Marayes y Chepes, provincias de San Juan y La Rioja, Argentina). Se realizaron cortes transversales, se observaron con microscopio óptico y se obtuvieron imágenes digitales que se analizaron con el programa ImageJ. Los resultados indican que los vasos son pequeños, numerosos y distribuidos de manera diagonal; el parénquima axial es de tipo apotraqueal difuso. Asimismo, se observó que hacia los sitios más áridos, el área total de fibras aumenta significativamente y el área de parénquima disminuye. Por otro lado, el área total de vasos no presentó cambios, mientras que el grosor de pared de fibra aumentó levemente con el incremento de la aridez. Estos rasgos sugieren que $B$. retama posee un leño altamente tolerante a la sequía presentando plasticidad fenotípica a lo largo del gradiente de aridez, registrando además una madera xeromórfica de elevada densidad.

Palabras clave. Área en sección transversal; gradiente ambiental; rasgos xilemáticos; zonas áridas. 


\section{INTRODUCCIÓN}

El xilema secundario forma el leño de una planta y está estructurado principalmente por tres tipos celulares: vasos, parénquima y fibras, los que poseen diferentes funciones (Tyree \& Sperry, 1989; Baas et al., 2004; Martínez-Cabrera et al., 2009; Pratt \& Jacobsen, 2017). Los vasos están relacionados con la conducción del agua, las células parenquimáticas con el almacenamiento de agua y nutrientes y las fibras con el soporte y la resistencia mecánica a la aridez (Kozlowski, 1992; Hacke \& Sperry, 2001; Sperry, 2003; Tyree \& Zimmermann, 2013). La disposición y el área que ocupan cada uno de estos tipos celulares están relacionadas a las que ocupan los demás tipos celulares que componen al xilema secundario (Baas et al., 2004), asimismo la variación del área que ocupa cada tipo celular en respuesta a variables ambientales, permite incrementar o disminuir la tolerancia de las plantas frente a la aridez (Pratt \& Jacobsen, 2017).

Las especies de plantas que poseen amplias distribuciones geográficas a lo largo de gradientes ambientales, suelen reflejar modificaciones morfológicas o estructurales (Carlquist, 1977; Baas et al., 2004; Martínez-Cabrera et al., 2009) que permiten su supervivencia en los diferentes ambientes donde viven. Estos ajustes (plasticidad fenotípica) pueden ser estudiados a través de la variación conjunta de rasgos funcionales. Un rasgo funcional es cualquier característica morfo-fisiológica de un organismo que influye sobre su rendimiento y su adaptabilidad relativa o "fitness" (Violle et al., 2007). El grosor de pared de las fibras, el tipo de distribución, tamaño y número de vasos, y la proporción de fibras en relación a vasos y parénquima, son rasgos del xilema secundario (leño) que se pueden observar bajo cortes histológicos transversales y que son importantes para la supervivencia de plantas en ambientes áridos (Martínez-Cabrera et al., 2009). En este sentido, la variación conjunta de rasgos del xilema secundario a lo largo de un gradiente ambiental permitiría comprender el grado de plasticidad que presentan los rasgos leñosos de una planta y su potencial uso como predictores de tolerancia a la sequía o de distribución geográfica potencial.
Las plantas de zonas áridas presentan plasticidad fenotípica en varias estructuras y órganos relacionados con cambios en la disponibilidad de agua. Para el xilema secundario puede mencionarse la disminución del área y diámetro de vasos (Sperry et al., 2008; Hacke et al., 2017), y/o el aumento del grosor de pared y del área de fibras (Jacobsen et al., 2005; Martínez-Cabrera et al., 2009). El análisis del área del tipo celular parenquimático resulta más complejo, debido a que se han observado comportamientos diferentes de los parénquimas axial y radial frente a la aridez (Martínez-Cabrera et al., 2009), aumentando principalmente el parénquima axial con el incremento de la temperatura (Morris et al., 2016; Morris et al., 2018). La densidad del leño, por otra parte, es una propiedad clave en las plantas leñosas y es consecuencia de modificaciones de los rasgos mencionados anteriormente (Chave et al., 2009). Por otro lado, el índice de vulnerabilidad propuesto por Carlquist (1977), relaciona el diámetro medio del lumen de los vasos con el número de vasos. La densidad del leño y el índice de vulnerabilidad, generalmente son indicadores de la seguridad del xilema para conducir agua en ambientes con elevada aridez.

La variación de rasgos del xilema en gradientes de aridez ha sido ampliamente estudiada en plantas, entre especies (Martínez-Cabrera et al., 2009; Ziemińska et al., 2013), y dentro de una misma especie (García-Cervigón et al., 2018; Rosas et al., 2019), registrándose una relación entre el flujo de agua que puede ser transportado y la configuración del sistema hidráulico, lo que es influenciado por las condiciones ambientales (Tyree \& Zimmermann, 2013). La seguridad hidráulica del xilema explica el mantenimiento de la conductividad a medida que aumenta la aridez, disminuyendo la probabilidad de ocurrencia de cavitación. Por otro lado, la eficiencia hidráulica está determinada por la conductividad del xilema, que básicamente representa la inversión en vasos por unidad de área (Sperry et al., 2008). Eficiencia y seguridad son consideradas condiciones fundamentales en el funcionamiento del sistema hidráulico de la planta, y se ha observado que variaciones en las 
condiciones de aridez pueden producir cambios en la estructura del xilema de las plantas leñosas (Lindorf, 1994; Pratt \& Jacobsen, 2017; García-Cervigón et al., 2018), produciendo un incremento en la inversión de un tipo celular en detrimento de otro.

Bulnesia retama (Gillies ex Hook. et Arn.) Griseb. (retamo) pertenece a la familia Zygophyllaceae y se distribuye en una amplia región árida de Argentina (Porter, 1974), en las Provincias Fitogeográficas del Monte y del Chaco Árido (Cabrera, 1994). Ocupa hábitats muy secos tales como laderas pedregosas en zonas con precipitaciones menores a los $100 \mathrm{~mm}$ anuales en el N de Mendoza y S de San Juan. Es un elemento característico de las provincias de Catamarca, La Rioja, San Juan, Mendoza, oeste de Córdoba, Santiago del Estero, San Luis y La Pampa, entre 500 y 2500 m s.m. (Hunziker, 1994). Se encuentra formando matorrales puros, dominando arbustales junto a bosques bajos de algarrobo dulce (Prosopis flexuosa DC.), o formando parte de arbustales mixtos junto a especies del género Larrea (Morello, 1958). También abunda en un reducido enclave en los semidesiertos de Ica, Ingenio y Nazca en Perú, encontrándosela a lo largo de conos de deyección, torrentes secos y riachos subterráneos que bajan de la cordillera, en una zona en la que prácticamente no hay precipitaciones; esto sugiere que en ciertos ambientes podría comportarse como una especie freatófita (Palacios \& Hunziker, 1984).

El retamo es un arbusto o pequeño árbol multicaulinar, de 1,5 a 3 metros de altura, si bien en algunos casos puede alcanzar los 6 metros y desarrollar tallos de hasta $35 \mathrm{~cm}$ de diámetro o mayores (Tortorelli, 1956). Los tallos y ramas están cubiertos por ceras que les dan color verde grisáceo, mientras que las hojas son efímeras (Palacios \& Hunziker, 1984). Su madera es extremadamente dura y su porción más interna está infiltrada con una resina de goma perfumada que le da a la madera un aspecto y tacto aceitosos (Record, 1921). Los retamales se usaron históricamente como recurso forestal, para confeccionar postes de viñedos, corrales, también para tornería y extracción de cera (Dalmasso \& Llera, 1996; Sosa \& Fernández, 1998).
Bulnesia retama es una especie que se ha adaptado al aumento progresivo de las condiciones de aridez en el continente, hacia fines del Oligoceno, principios del Mioceno (Godoy-Bürki et al., 2018). Como especie de ambientes áridos es altamente tolerante a la sequía (Gatica et al., 2017), presentando valores de potenciales agua en un rango entre -4 y -7,5 MPa (Ribas-Fernández, 2016).

Se han estudiado aspectos taxonómicos $\mathrm{y}$ evolutivos de la especie B. retama (Crisci et al., 1979; Comas \& Hunziker, 1996; Godoy-Bürki et al., 2018), su biología reproductiva (Debandi et al., 2002), la fisiología de la germinación (Rodríguez-Rivera et al., 2007), el destino de sus semillas desde la dispersión primaria hasta la emergencia de plántulas y el establecimiento (Ribas-Fernández et al., 2009), sin embargo existen escasos trabajos disponibles (Record, 1921; Böcher \& Lyshede, 1968), y otros de difícil acceso (Cozzo, 1948; Tortorelli, 1956), sobre los rasgos del xilema secundario que presenta esta especie. Asimismo, no existen trabajos que estudien variaciones en rasgos del xilema secundario a lo largo de gradientes de aridez para esta especie. Los objetivos de este trabajo son: i) describir anatómicamente, a través de cortes histológicos transversales, el xilema secundario de B. retama; ii) analizar la variación del porcentaje de vasos, fibras y parénquima, y grosor de la pared de fibra; iii) analizar si la modificación en el área que ocupa un tipo celular dentro del xilema secundario implica la reducción o aumento del área de otro tipo celular; y iv) analizar la seguridad hidráulica de la madera de $B$. retama frente a la aridez a través del valor de densidad específica del leño y del índice de vulnerabilidad de Carlquist (1977), a lo largo de un amplio gradiente de aridez en la zona central-oeste de Argentina.

\section{MATERIALES Y MÉTODOS}

\section{Sitios de estudio}

Los cuatro sitios de estudio se ubicaron a lo largo de un gradiente de precipitaciones con sentido Oeste-Este de más de $200 \mathrm{~mm}$ anuales, entre las provincias de San Juan (Médanos Grandes, Bermejo y Marayes) y La Rioja (Chepes), abarcando poco más de $200 \mathrm{~km}$ (Fig. 1). 

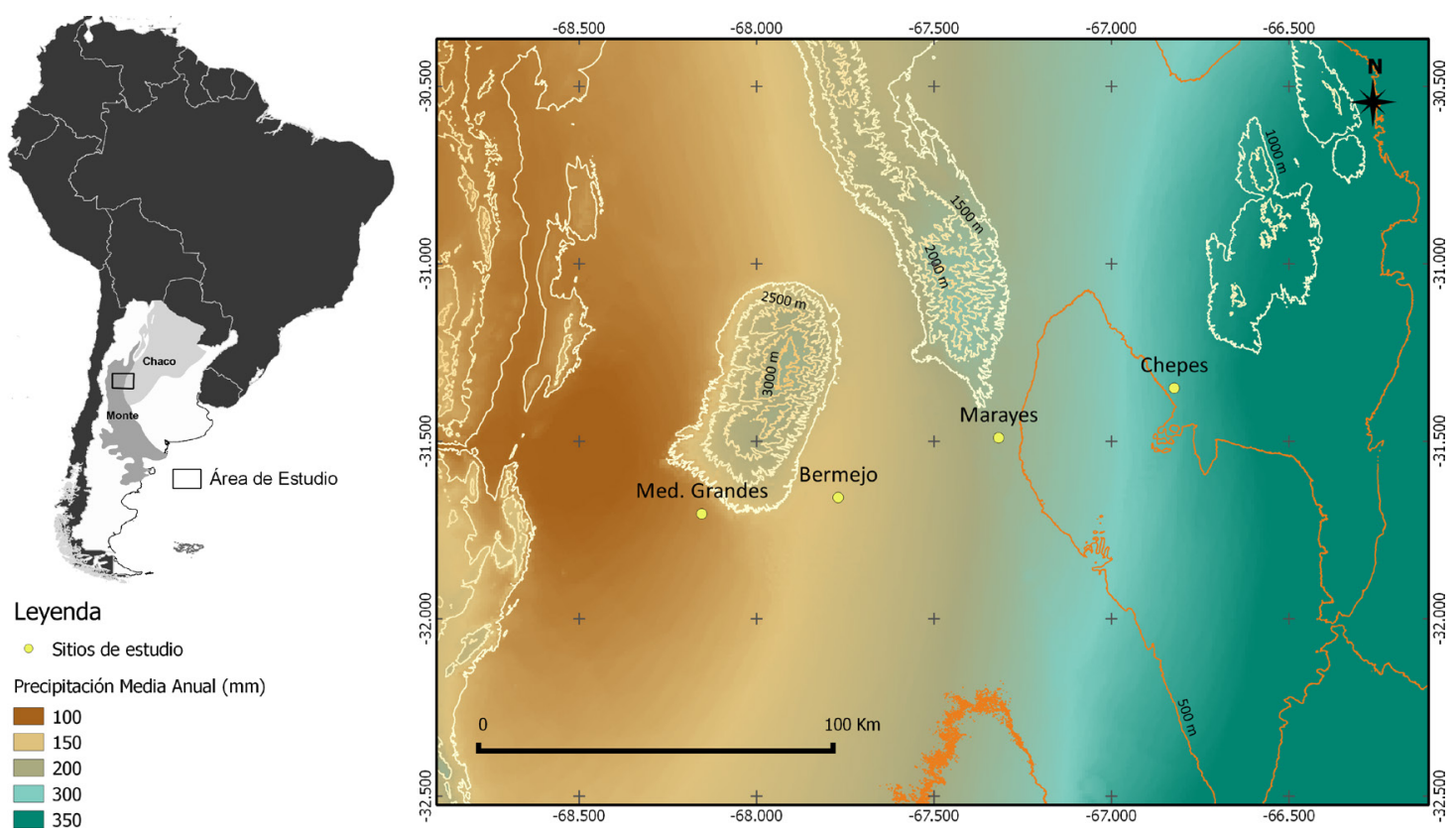

Fig. 1. Localización de los sitios estudiados que conforman el gradiente de aridez en el centro-oeste de Argentina, Médanos Grandes, Bermejo y Marayes (San Juan) y Chepes (La Rioja). Imagen realizada mediante el programa QGIS (QGIS Development team, 2020). Datos de precipitación media anual tomados de la base de datos global WorldClim (Hijmans et al., 2005). Figura en color en la versión en línea http:/www.ojs.darwin.edu.ar/index.php/darwiniana/article/view/929/1215

La temperatura media anual se mantiene prácticamente estable en todo el gradiente, con alrededor de $18{ }^{\circ} \mathrm{C}$. Las características climáticas de los cuatro sitios varían desde muy áridas al oeste, con una precipitación media anual (desde ahora PMA) de $130 \mathrm{~mm}$ en la zona de Médanos Grandes, hasta semiáridas al este, con PMA de $357 \mathrm{~mm}$ en la localidad de Chepes (Tabla 1). Desde el punto de vista fisonómico-florístico, el gradiente comprende las Provincias Fitogeográficas del Monte, en el extremo occidental, y del Chaco Árido, en el extremo más oriental (Abraham et al., 2009). En los sitios más áridos (Médanos Grandes y Bermejo) la vegetación es típica de matorrales abiertos, dominados por arbustos del género Larrea (L. divaricata Cav. y L. cuneifolia Cav.), acompañados por B. retama y Atamisquea emarginata Miers ex Hook. \& Arn.; mientras que en los sitios que se encuentran dentro de la Provincia Fitogeográfica del Chaco Árido (Marayes y Chepes), la vegetación es un algarrobal abierto, con individuos dispersos de Prosopis flexuosa, menor abundancia de $B$. retama, Parkinsonia praecox (Ruiz \& Pav. ex Hook.) Hawkins y Aspidosperma quebracho-blanco Schltdl. (Gatica, 2015).

\section{Obtención y preparación de las muestras}

Para cada sitio se seleccionaron 10 individuos adultos, aparentemente sanos y similares en tamaño (2,5 $\mathrm{m}$ de altura en promedio). A partir de una rama primaria de cada individuo ( $\geq 5 \mathrm{~cm}$ de diámetro), se extrajo una muestra de aproximadamente $10 \mathrm{~cm}$ de largo para analizar en el laboratorio (10 individuos $\mathrm{x}$ 4 sitios $=40$ muestras). Para la selección de las ramas no se tuvo en cuenta su orientación. De cada muestra se obtuvo una submuestra de 2,5 $\mathrm{cm}$ de largo, la cual se cortó longitudinalmente en dos mitades, una para realizar los preparados histológicos y otra para estimar la densidad del leño.

Para la obtención de los preparados histológicos, se colocaron cubos de leño de $1 \mathrm{~cm}^{3}$ en FAA $(10 \%$ formaldehído, $5 \%$ ácido acético glacial, $50 \%$ etanol y $35 \%$ agua destilada) con la finalidad de ablandar los tejidos y fijar las muestras. Luego, los cubos se llevaron a punto de ebullición, alrededor de 10 horas diarias durante una semana, en recipientes con agua y dos gotas de detergente. Con un micrótomo de deslizamiento Leica SM2000R se realizaron cortes transversales de $5 \mu \mathrm{m}$ de espesor. Se seleccionaron 
Tabla 1. Características ambientales y bióticas de cuatro sitios analizados a lo largo de un gradiente de aridez en el centrooeste de Argentina. Para el cálculo de índice de aridez junto con la evapotranspiración potencial, se utilizó la base de datos global CGIAR-CSI (https://cgiarcsi.community/data/global-aridity-and-pet-database/). La precipitación media anual y la temperatura media anual de cada sitio se obtuvieron de la base de datos global WorldClim para el período 1950-2000 (Hijmans et al., 2005). La riqueza de especies y la cobertura de la vegetación se calcularon a partir de Gatica (2015).

\begin{tabular}{|c|c|c|c|c|}
\hline & \multicolumn{4}{|c|}{ Sitios } \\
\hline & Médanos Grandes & Bermejo & Marayes & Chepes \\
\hline Latitud (Sur) & $31^{\circ} 42^{\prime} 55,44^{\prime \prime}$ & $31^{\circ} 39^{\prime} 30,74^{\prime \prime}$ & $31^{\circ} 29^{\prime} 19,97^{\prime \prime}$ & $31^{\circ} 21^{\prime} 1,12^{\prime \prime}$ \\
\hline Longitud (Oeste) & $68^{\circ} 8^{\prime} 47,44^{\prime \prime}$ & $67^{\circ} 46^{\prime} 12,94^{\prime \prime}$ & $67^{\circ} 19^{\prime} 0,59^{\prime \prime}$ & $66^{\circ} 49^{\prime} 24,17^{\prime \prime}$ \\
\hline $\begin{array}{l}\text { Índice de aridez (precipitación media } \\
\text { anual/evapotranspiración potencial) }\end{array}$ & 0,08 & 0,12 & 0,17 & 0,23 \\
\hline $\begin{array}{l}\text { Precipitación media anual } \\
\text { (coeficiente de variación) en mm }\end{array}$ & $130(79 \%)$ & $188(86 \%)$ & $262(84 \%)$ & $357(85 \%)$ \\
\hline $\begin{array}{l}\text { Temperatura media anual } \\
\text { (desvío estándar) en }{ }^{\circ} \mathrm{C}\end{array}$ & $17,7( \pm 6,2)$ & $18,1( \pm 6,1)$ & $18,8( \pm 5,8)$ & $19,2( \pm 5,6)$ \\
\hline Riqueza de especies & $4( \pm 0,82)$ & $4,2( \pm 0,96)$ & $6,5( \pm 1,73)$ & $12,7( \pm 1,53)$ \\
\hline Cobertura de la vegetación $(\%)$ & $31,17( \pm 3,44)$ & $42,35( \pm 11,4)$ & $61,82( \pm 11,3)$ & $96,53( \pm 3,46)$ \\
\hline Estructura de la vegetación & Matorral & Arbustal xerófilo & Arbustal xerófilo & Bosque xerófilo \\
\hline Altitud (m s.m.) & 600 & 569 & 571 & 654 \\
\hline
\end{tabular}

los mejores cortes, se deshidrataron mediante una serie de etanol $(70 \%, 90 \%$ y $100 \%)$, se clarificaron con hipoclorito de sodio al $10 \%$ y se tiñeron bajo la técnica de tinción sucesiva doble de Safranina-Fast Green. El montaje de los preparados se realizó con Bálsamo de Canadá (D'ambrogio de Argüeso, 1986; Ruzin, 1999). Sobre la base de estudios previos, se realizaron solamente cortes transversales ya que este tipo de corte es el óptimo para estudiar cambios frente a condiciones ambientales en las áreas que ocupan los distintos tipos celulares que forman el xilema secundario, como así también cambios en el diámetro de vasos y paredes de fibras (Carlquist, 1988; Villar-Salvador et al., 1997; Martínez Cabrera et al., 2009; Ziemińska et al., 2013; Hajek et al., 2014; Ziemińska et al., 2015; Morris et al., 2016; Pratt \& Jacobsen 2017). Los preparados histológicos se realizaron en el laboratorio de anatomía vegetal del Instituto Argentino de Nivología, Glaciología y Ciencias Ambientales (IANIGLA-CONICET), Provincia de Mendoza y se encuentran resguardados en el laboratorio del Grupo de Ecología del Desierto (GEDes), del Departamento de Biología de la Facultad de Ciencias Exactas, Físicas y Naturales de la Universidad Nacional de San Juan, Argentina.
Se obtuvieron imágenes a diferentes aumentos $(40 \mathrm{x}, 100 \mathrm{x}$ y $400 \mathrm{x})$ mediante microscopio con cámara incorporada (digital biological microscope model DMWB1-223). Se tomaron 5 imágenes de cada corte transversal en diferentes sectores de los anillos de crecimiento más externos (Martínez-Cabrera et al., 2009). Para cada corte transversal, se registró tipo de anillo de crecimiento, disposición de vasos, diámetro medio de lumen de vasos, tipo de fibras según la comparación del diámetro de lumen con el grosor de la pared, grosor de la pared de fibra y tipo de parénquima axial. Para la descripción de estas variables del xilema secundario, se siguió la terminología del comité de Nomenclatura de IAWA (IAWA, 1989). El diámetro de los vasos se consideró muy pequeño $(<50 \mu \mathrm{m}$, categoría $\mathrm{I})$, pequeño $(51 \leq 100 \mu \mathrm{m}$, categoría II), mediano $(101 \leq 200 \mu \mathrm{m}$, categoría III) y grande $(>201 \mu \mathrm{m}$, categoría IV), según lo establecido por el comité de IAWA (1989) y por Wheeler \& Baas (1998).

Para analizar el porcentaje ocupado por cada tipo celular que forma el xilema secundario (parénquima, vasos y fibras) se utilizó el método de estimación de la cobertura porcentual por puntos de muestreo propuesto por Smith(1967), con algunas modificaciones. 
Esto consiste en instalar una grilla aleatoria sobre la imagen en donde la totalidad de los puntos representan el $100 \%$ y luego se cuenta la cantidad de puntos que caen sobre cada tipo celular. Posteriormente se obtiene el porcentaje de cada tipo celular multiplicando la cantidad de puntos afectados por 100 y dividiéndolo por la totalidad de puntos de la imagen. Para el tipo celular parenquimático se analizaron en conjunto el parénquima axial y los radios parenquimáticos. Todos los análisis se realizaron mediante el software libre ImageJ $1.52 \mathrm{a}$ (Rasband, 2018).

La densidad específica del leño se obtuvo según el protocolo de Pérez-Harguindeguy et al. (2013), la cual se define como el peso seco de la muestra de un tallo o rama sin la corteza (secada a $100{ }^{\circ} \mathrm{C}$ durante $72 \mathrm{hs}$ ), dividido por el volumen de la misma sección, cuando aún permanece fresco. El procedimiento para la obtención del volumen en fresco consiste en sumergir la muestra completamente en agua destilada en un vaso de precipitación con la ayuda de una aguja o pinza. Al sumergir la muestra, el nivel de agua aumenta incrementando el peso que es registrado por la balanza (el peso del agua desplazada), que equivale al volumen de la muestra en $\mathrm{cm}^{3}$ (ya que el agua tiene una densidad de $\left.1 \mathrm{~g} . \mathrm{cm}^{-3}\right)$. Si bien la densidad de leño varía dentro de cada parte de la planta (Chave et al., 2009), existen antecedentes de que las ramas presentan una densidad menor de aproximadamente $9 \%$ del valor de densidad del leño del tallo (Chave, 2005; Sarmiento et al., 2011). También diversos trabajos han mostrado que, si bien existen algunas diferencias con el tallo, las ramas son de utilidad para estudios sobre características y variación de los tipos celulares que forman el xilema frente a condiciones climáticas (Villar-Salvador et al., 1997; Davis et al., 1999; Ziemińska et al., 2013, 2015; Hajek et al., 2014; Morris et al., 2016; Pratt \& Jacobsen, 2017; Kiorapostolou et al., 2019).

Se calculó el índice de vulnerabilidad de Carlquist (1977) para determinar el grado de xeromorfismo de la madera de $B$. retama. Este índice se basa en la relación diámetro medio del lumen de vasos sobre el número de vasos por área $\left(\mathrm{mm}^{2}\right)$. Valores mayores a 1 de este índice, indican una madera mesomórfica, y valores menores 1 indican una madera xeromórfica, con características relacionadas a una conducción más segura del agua en condiciones de elevada aridez.
Para calcular el índice de aridez junto con la evapotranspiración potencial, se utilizó la base de datos global de índice de aridez y evapotranspiración CGIAR-CSI (https://cgiarcsi. community/data/global-aridity-and-pet-database/). Para evaluar el efecto de la aridez (índice de aridez, variable regresora) sobre las variaciones que presentan los porcentajes de área de vasos, parénquima y fibras, además del grosor de pared de fibra, densidad de leño e índice de vulnerabilidad (variables respuesta), se ajustaron modelos de regresión lineal $(\mathrm{lm})$, considerando todas las variables respuesta transformadas a logaritmo para cumplir supuestos de normalidad (evaluado a través del paquete fitdistrplus, Delignette-Muller \& Dutang, 2015). Por otro lado, se evaluó la existencia de compromisos (la pérdida o ganancia de un tipo celular respecto de otro) entre los rasgos de área de los tipos celulares que conforman al leño (vasos, parénquima y fibras) por medio de correlaciones de Pearson. Se consideraron compromisos sólo cuando los valores de correlación fueran negativos y significativos. Para la totalidad de las pruebas estadísticas utilizamos el programa estadístico $\mathrm{R}$ versión 3.5.1 (R CoreTeam, 2018).

\section{RESULTADOS}

En el xilema secundario de B. retama se observaron anillos visibles, caracterizados por presentar cambios anatómicos graduales, debido a que los vasos pueden continuar a través de dos o tres anillos. Su porosidad es de tipo semi-circular, con vasos predominantemente solitarios (en ocasiones agrupados) con disposición de tipo dendrítica (Fig. 2A). El análisis de los tipos celulares mostró que las fibras presentan paredes gruesas, de 1,8-5,7 $\mu \mathrm{m}$ de espesor (valor medio $3,13 \mu \mathrm{m}$ ), con un diámetro del lumen pequeño en relación a la pared, por lo que forman parte de la categoría de fibras de paredes muy gruesas (Tabla 2). Por otro lado, B. retama presentó un parénquima axial de tipo apotraqueal difuso, en ocasiones formando hebras de parénquima único o pares de hebras distribuidos entre los elementos fibrosos del leño, sugiriendo la presencia de parénquima apotraqueal difuso en agregados (Fig. 2B). El diámetro de los vasos registró un valor de 19,55-40,34 $\mu \mathrm{m}$ (valor medio de 27,34 $\mu \mathrm{m}$ ), 

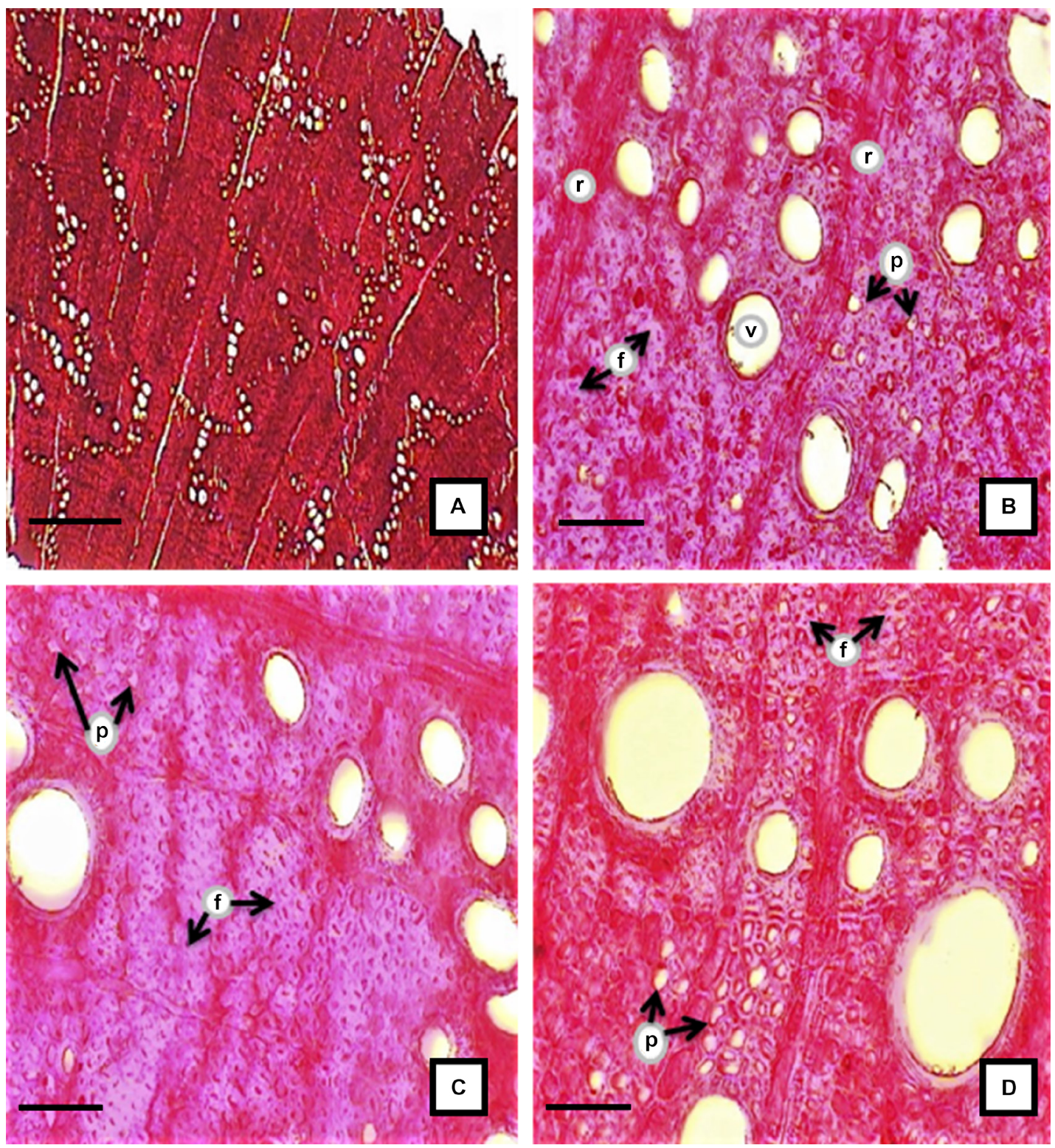

Fig. 2. Cortes transversales de leño de Bulnesia retama de individuos colectados a lo largo de un gradiente de aridez del centrooeste de Argentina. A, porosidad semi-circular y disposición dendrítica de vasos. B, detalle de vasos, fibras de paredes muy gruesas y parénquima axial apotraqueal. C, leño de un ejemplar del sitio más árido (Médanos Grandes), con gran predominio de fibras. D, leño de un ejemplar perteneciente del sitio menos árido (Chepes), con mayor predominio de células de parénquima. Abreviaturas: f, fibras; r, radios parenquimáticos; v, vasos; p, parénquima axial. Escalas: A, $500 \mu \mathrm{m} ; \mathrm{B}, 50 \mu \mathrm{m} ; \mathrm{C}, 50 \mu \mathrm{m}$; D, $50 \mu \mathrm{m}$. Figura en color en la versión en línea http://www.ojs.darwin.edu.ar/index.php/darwiniana/article/view/929/1215

correspondiente a la clasificación de vasos muy pequeños (categoría I de IAWA). Los vasos ocuparon un promedio del 18,13\% (9,5-33,3\%) del xilema secundario. El parénquima (axial+radial) ocupó un promedio del 30,19\% (13,3-51,4\%). Las fibras ocuparon, en promedio, un $51,68 \%$ $(28,6-73,3 \%)$. En la Tabla 3 se indica la estadística descriptiva de las variables estudiadas. 
Tabla 2. Listado de rasgos anatómicos del xilema secundario observados en individuos de $B$. retama colectados en cuatro sitios a lo largo de un gradiente de aridez en el centro-oeste de Argentina.

\begin{tabular}{|c|c|}
\hline Porosidad & Semi-circular \\
\hline Disposición de vasos & Dendrítica \\
\hline Tamaño de vasos & $\begin{array}{c}\text { Muy pequeños } \\
\text { (Categoría I IAWA) }\end{array}$ \\
\hline Tipo de fibras & De paredes muy gruesas \\
\hline Tipo de parénquima axial & Apotraqueal difuso \\
\hline
\end{tabular}

En relación con el gradiente de aridez, si bien se observó una leve disminución en la media de los porcentajes de área de vasos (conducción) en relación a la aridez, esta variación no fue significativa $\quad\left(\mathrm{p}=0,16 ; \quad R^{2}=0,02 ; \quad\right.$ Fig. $\left.3 \mathrm{~A}\right)$, presentando una gran variabilidad entre los individuos de cada sitio. Por otro lado, se registraron cambios significativos en el área del xilema secundario ocupada por las fibras (soporte y resistencia) y el parénquima (almacenamiento) (Fig. 3B y C). El área ocupada por fibras mostró un fuerte aumento hacia el sector más árido del gradiente ( $\mathrm{p}<0,0001 ; R^{2}=0,70$; Fig. $\left.3 \mathrm{~B}\right)$, mientras que el área de parénquima disminuyó con la aridez $\left(\mathrm{p}<0,0001 ; R^{2}=0,71 ;\right.$ Fig. $\left.3 \mathrm{C}\right)$. Además, si bien no se encontró un buen ajuste entre el grosor de la pared de fibras y la aridez, éste presentó un aumento significativo hacia la porción más árida del gradiente ( $\mathrm{p}=0,04 ; R^{2}=0,07$; Fig. 3D).

$\mathrm{Se}$ registraron compromisos significativos mediante el análisis de correlaciones de Pearson entre los rasgos de área de fibras-parénquima y fibras-vasos para todo el gradiente. Según los valores de correlación, se observó que el compromiso en la inversión de fibras aumenta hacia la porción más árida del gradiente en detrimento de los vasos y del parénquima, siendo más pronunciado el compromiso entre vasos y fibras en los sitios de mayor aridez. No se observaron compromisos entre vasos y parénquima en ningún sitio a lo largo del gradiente (Tabla 4).

En relación a los indicadores de seguridad hidráulica del xilema secundario de $B$. retama, no se registraron variaciones significativas en la densidad del leño $(\mathrm{p}=0,42)$, como tampoco en el índice de vulnerabilidad de Carlquist $(\mathrm{p}=0,74)$ a lo largo del gradiente. A pesar de no presentar variaciones significativas, la propiedad de densidad específica de leño registró un elevado valor promedio de 0,929 $\mathrm{gr} / \mathrm{cm}^{3}$. Por otro lado, el índice de vulnerabilidad de Carlquist (1977) presentó un valor promedio de 0,7; este valor es característico de leños xeromórficos, seguros en la conducción de agua.

\section{DISCUSIÓN}

La porosidad semi-circular que se registró en $B$. retama concuerda con lo registrado para la misma especie por Tortorelli (1956) y con la especie cercana Larrea nitida Cav. (Andreoni, 2014). Por otro lado, B. retama difiere de la porosidad difusa que presenta Bulnesia sarmientoi Lorentz ex Griseb. (Giménez et al. 2007), pero comparte el patrón dendrítico de los vasos. La porosidad semi-circular coincide además con lo observado en especies de grupos taxonómicos distantes con las que $B$. retama comparte hábitat en el Monte, como Prosopis alba Griseb. y Geoffroea decorticans (Gillies ex Hook. \& Arn.) Burkart (Barañao et al., 2008). Finalmente, se observó que $B$. retama presenta la peculiar disposición de los vasos registrada por Böcher \& Lyshede (1968), esto es, que los vasos no se limitan a anillos anuales únicos, sino que pueden continuar a través de dos o tres anillos.

El análisis del xilema secundario de $B$. retama observado en cortes histológicos transversales mostró que presenta gran cantidad de fibras de paredes gruesas y vasos principalmente solitarios, coincidiendo estas características con las especies cercanas $L$. divaricata, $L$. cuneifolia y $L$. nítida (Andreoni, 2014), y solamente se asemeja en el tipo de fibras con Bulnesia sarmientoi (Burgerstein, 1912). Además, se registraron características del xilema secundario coincidentes con lo observado por Tortorelli (1956), como vasos muy pequeños y numerosos dispuestos de manera dendrítica y un parénquima axial de tipo apotraqueal difuso y probablemente de tipo difuso en agregados.

Del análisis de vasos, se infiere que $B$. retama presenta un sistema hidráulico con vasos numerosos y pequeños, característica típica de especies adaptadas a la aridez, donde el caudal 


\section{E. A. MELIÁN ET AL. Variación de rasgos del xilema secundario de Bulnesia retama}

Tabla 3. Valor promedio y desvío estándar (entre paréntesis) de rasgos del leño, densidad de leño e índice de vulnerabilidad (Carlquist, 1977), estudiados en individuos ( $\mathrm{n}=10)$ de B. retama en cuatro sitios a lo largo de un gradiente de aridez en el centro-oeste de Argentina.

\begin{tabular}{llllll}
\hline & \multicolumn{5}{c}{ Sitios } \\
Variables analizadas & Médanos Grandes & Bermejo & Marayes & Chepes & Total Gradiente \\
\hline Área de vasos $(\%)$ & $16,91( \pm 6,02)$ & $16,68( \pm 4,38)$ & $19,43( \pm 4,29)$ & $19,51( \pm 6,35)$ & $18,13( \pm 5,31)$ \\
Área de fibras (\%) & $62,3( \pm 6,61)$ & $64,95( \pm 6,02)$ & $43,85( \pm 7,22)$ & $35,61( \pm 5,5)$ & $51,68( \pm 13,91)$ \\
Área de parénquima $(\%)$ & $20,79( \pm 3,1)$ & $18,37( \pm 3,03)$ & $36,72( \pm 6,79)$ & $44,88( \pm 5,46)$ & $30,19( \pm 12,11)$ \\
$\begin{array}{l}\text { Diámetro de vasos }(\mu \mathrm{m}) \\
\text { Número de vasos/mm } / \mathrm{mm}^{2}\end{array}$ & $29,74( \pm 5)$ & $23,36( \pm 2)$ & $30,76( \pm 6)$ & $25,48( \pm 3)$ & $27,33( \pm 5)$ \\
$\begin{array}{l}\text { Grosor de pared de fibra }(\mu \mathrm{m}) \\
\text { Densidad de leño }\left(\mathrm{gr} / \mathrm{cm}^{3}\right)\end{array}$ & $3,68( \pm 1,1)$ & $2,87( \pm 0,35)$ & $3,12( \pm 0,77)$ & $2,85( \pm 0,57)$ & $3,13( \pm 0,8)$ \\
$\begin{array}{l}\text { Índice de vulnerabilidad } \\
\text { (diámetro medio de }\end{array}$ & $0,927( \pm 0,002)$ & $0,932( \pm 0,001)$ & $0,931( \pm 0,001)$ & $0,927( \pm 0,002)$ & $0,929( \pm 0,003)$ \\
vaso/vasos.mm $\left.{ }^{-2}\right)$ & 0,9 & 0,4 & 0,7 & 0,7 & 0,7 \\
\hline
\end{tabular}

es restringido, asegurando el transporte del agua escasa (Carlquist, 1988; Rosell et al., 2017). Debido a que a este tipo de leño se relaciona con la prevención del colapso bajo tensiones muy negativas o temperaturas muy bajas, se considera como una estrategia que poseen ciertas plantas leñosas para una conducción segura del agua en zonas áridas (Tyree \& Zimmermann, 2013). Esto se observó en especies cercanas a $B$. retama como Larrea nitida y Larrea tridentata (DC.) Coville (Medeiros \& Pockman, 2014).

En este trabajo no se registraron cambios evidentes en el área de vasos asociados con el aumento de la aridez, si bien esto podría ser considerado un patrón general en plantas de zonas áridas (Maherali et al., 2004; Martínez-Cabrera et al., 2009), es posible que su probable condición de planta freatófita en ciertos ambientes esté influyendo sobre la falta de variación de este rasgo, como lo sugiere Hunziker (1994). En cambio, sí se registró una gran variabilidad en el área de vasos entre individuos dentro de un mismo sitio. Esto podría ser explicado por la distinta orientación de las ramas que fueron analizadas en cada individuo, ya que se ha observado en especies de arbustos de ambientes áridos que el transporte del agua está segmentado dentro de la misma planta (Schenk et al., 2008), pudiendo tener influencia la pendiente, cercanía a un curso de agua, etc.
El leve aumento del grosor de la pared de fibras registrado hacia los sitios más áridos se puede interpretar como un aumento en la resistencia mecánica frente a los efectos de la aridez, en concordancia con lo que observaron Jacobsen y colaboradores (2005) en seis especies de arbustos de las familias Rosaceae, Rhamnaceae y Anacardiaceae del sur de California. Además, es probable que las fibras compensen la disminución de parénquima en los sitios más áridos, debido a que se ha observado que éstas pueden almacenar carbohidratos sin comprometer su capacidad mecánica (Pratt \& Jacobsen, 2017).

El parénquima, el segundo tipo celular más abundante en el leño de $B$. retama, probablemente compense el bajo porcentaje ocupado por los vasos, sobre todo en el extremo más árido del gradiente, debido a que el parénquima puede cumplir funciones de almacenamiento de agua, además de recuperación de vasos afectados por embolia (Morris et al., 2016; Klein et al., 2018). La seguridad del xilema secundario es de gran interés debido a su papel en la resistencia a la aridez de las plantas (Choat et al., 2012; Sperry \& Love, 2015). Junto con la seguridad, la eficiencia y las compensaciones mecánicas, el almacenamiento es una función crítica del leño y está asociado con muchos de los aspectos dinámicos de su función (Pratt \& Jacobsen, 2017). 

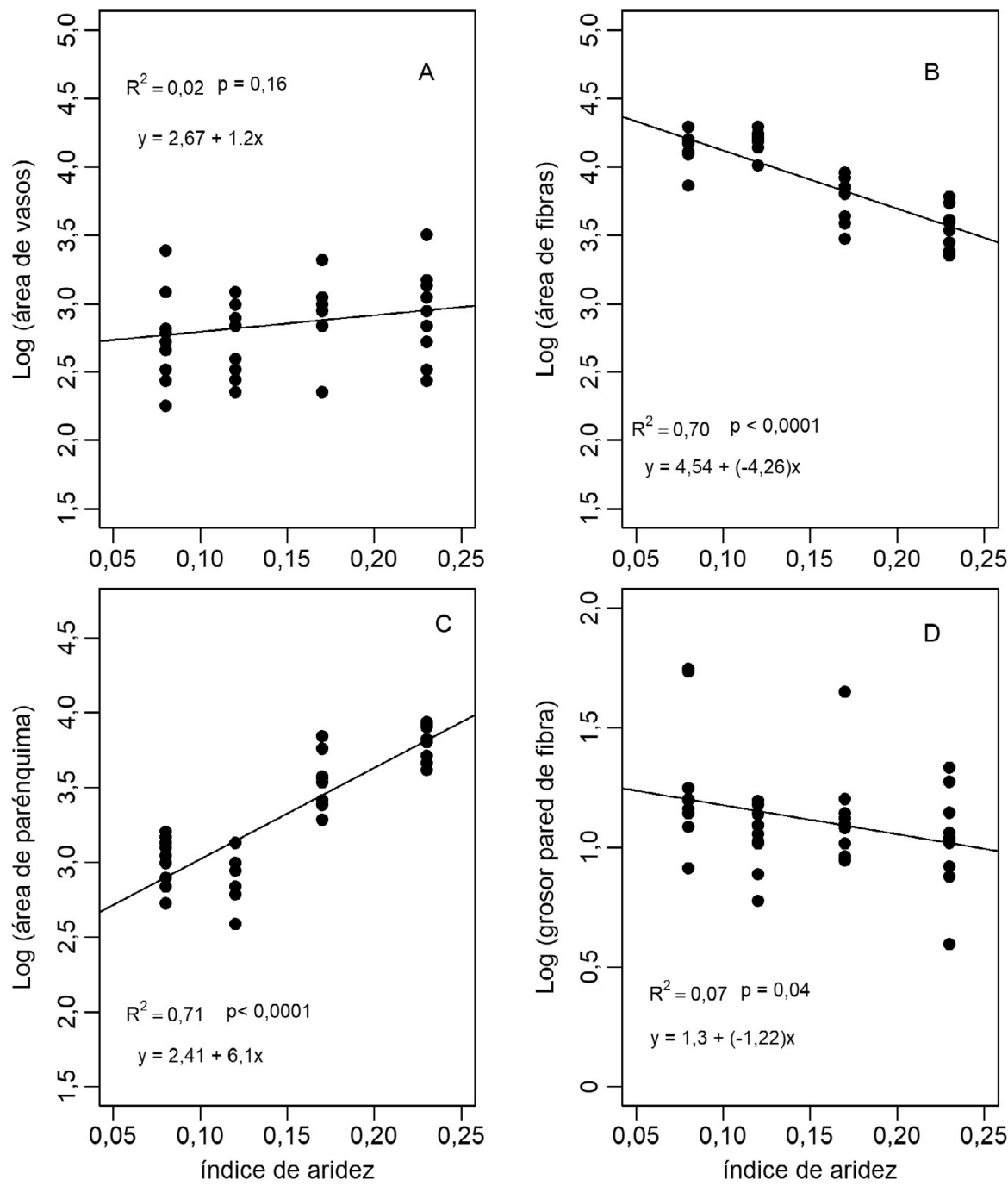

Fig. 3. Variación de rasgos del leño de Bulnesia retama a lo largo de un gradiente de aridez, en el centro-oeste de Argentina. A, disminución no significativa del área de vasos hacia el sector más árido del gradiente. B, aumento significativo del área de fibras hacia el sector más árido del gradiente. $\mathbf{C}$, disminución significativa del área de parénquima hacia el sector más árido del gradiente. D, aumento marginalmente significativo del grosor de la pared de fibra hacia el sector más árido del gradiente. Para analizar la variación de estos rasgos a lo largo del gradiente de aridez se ajustaron modelos de regresión lineal $(\mathrm{lm})$, considerando todas las variables respuesta transformadas a logaritmo para cumplir supuestos de normalidad.

Los compromisos asociados con el almacenamiento de carbohidratos pueden estar relacionados con el empaquetamiento espacial, ya que si el leño está ocupado con mayor número de células parenquimáticas, entonces tendrá menos volumen para las fibras y vasos. Se ha observado que el aumento en el contenido de parénquima se asocia negativamente con el contenido de fibras pero es independiente del contenido de vasos, como registraron Ziemińska y colaboradores (2015) en 69 especies de árboles y arbustos de Australia. Probablemente esto explica que el compromiso entre vasos y parénquima no sea significativo en nuestro análisis. También las fibras resultaron ser más abundantes en los sitios más áridos del gradiente. 
Tabla 4. Correlaciones de Pearson (r) entre las áreas de vasos, fibras y parénquima por sitios y valores medios totales del gradiente para analizar compromisos (pérdida o ganancia de un tejido respecto de otro) dentro del xilema secundario de B. retama, a lo largo de un gradiente de aridez en el centro-oeste de Argentina (Tabla 1). En negrita las correlaciones significativas $* \mathrm{p}=0,05 ; * * \mathrm{p}<0,05 ; * * * \mathrm{p}<0.0001$.

\begin{tabular}{llllll}
\hline & \multicolumn{3}{c}{ Sitios } \\
Compromisos entre tejidos & Médanos Grandes & Bermejo & Marayes & Chepes & Total Gradiente \\
\hline Fibras-Vasos & $\mathbf{- 0 , 8 8} * *$ & $\mathbf{- 0 , 8 8} * *$ & $-0,39$ & $-0,58$ & $\mathbf{- 0 , 5 1} * *$ \\
Fibras-Parénquima & $-0,41$ & $\mathbf{- 0 , 7 2} * *$ & $\mathbf{- 0 . 8 1} * *$ & $-0,33$ & $\mathbf{- 0 , 9 2} * * *$ \\
Vasos-Parénquima & $-0,06$ & 0,29 & $-0,21$ & $-0,57$ & 0,15
\end{tabular}

Esto podría interpretarse como un reflejo de las elevadas tensiones a la que estaría sometido el xilema de $B$. retama en las épocas más secas, debido a que son un tipo celular relacionado con el soporte y la resistencia mecánica del xilema a la sequía. Las características de las fibras adyacentes a los vasos son importantes para prevenir su colapso bajo tensiones muy negativas (Jacobsen et al., 2005).

La densidad promedio del leño de $B$. retama $\left(0,929 \mathrm{gr} / \mathrm{cm}^{3}\right)$ registrada en este estudio es ligeramente mayor a $\operatorname{los} 0,800$ a $0,900 \mathrm{gr} / \mathrm{cm}^{3}$ descriptos por Bertonatti (1997) para esta misma especie. Este resultado indica que el leño de $B$. retama es de alta densidad si se tiene en cuenta que los valores de especies de ambientes áridos generalmente oscilan entre $0,600-1 \mathrm{~g} / \mathrm{cm}^{3}$, y que se consideran maderas de alta densidad aquéllas que superan los $0,751 \mathrm{gr} / \mathrm{cm}^{3}$ (Giménez \& Moglia, 2003). Comparando con otras especies que habitan las Provincias Fitogeográficas del Monte y del Chaco Árido, también muy utilizadas por su densa madera, los valores de densidad de leño encontrados en $B$. retama fueron superiores a Geoffroea decorticans $\left(0,600 \mathrm{gr} / \mathrm{cm}^{3}\right)$, Prosopis alba $\left(0,800 \mathrm{gr} / \mathrm{cm}^{3}\right)$, Celtis tala Gillies ex Planch. $\left(0,800 \mathrm{gr} / \mathrm{cm}^{3}\right)$ y Vachellia caven (Molina) Seigler \& Ebinger $\left(0,890 \mathrm{gr} / \mathrm{cm}^{3}\right)$ (Giménez \& Moglia, 2003). Posiblemente, estos elevados valores estén relacionados con sus vasos pequeños y la gran cantidad de fibra con paredes gruesas que se estructuran en la madera de $B$. retama, junto a las resinas y aceites que posee. La madera de B. retama no presenta problemas con su uso en comparación con otra especie muy utilizada por la dureza de su madera, el quebracho blanco (Aspidosperma quebracho-blanco). Esta última especie nativa, muy utilizada como recurso forestal, comparte la Provincia Fitogeográfica del Chaco Árido con B. retama, posee una densidad de leño de $0,885 \mathrm{gr} / \mathrm{cm}^{3}$ y, aunque puede responder bien al curvado y golpes, tiene tendencia a sufrir contracciones, deformaciones y roturas (Moglia et al., 2009). Estos problemas no fueron detectados en la madera de $B$. retama (Dalmasso et al., 2014) lo que la hace también apropiada para su utilización como arbolado.

Los valores registrados del índice de vulnerabilidad confirman que es una especie con elevada seguridad en la conducción hidráulica (valor promedio de 0,7 ) y que su leño posee características xeromórficas (Carlquist, 1977). Eso concuerda con el valor de diámetro de lumen muy pequeño, junto al gran número de vasos que registramos en este trabajo. Para otras especies de ambientes áridos subtropicales, se sugirió que la presencia de raíces profundas (plantas freatófitas, como posiblemente sea $B$. retama según Palacios \& Hunziker, 1984) puede derivar en características mesomórficas en el leño (Lindorf, 1994); sin embargo en B. retama registramos un leño de características xeromórficas. Bulnesia retama presentó un índice de vulnerabilidad menor a otra especie con cierto grado de afinidad taxonómica como es Guaiacum officinale L. $(3,16)$ (Lindorf, 1994). Por otro lado, comparando con otras especies con las que comparte su distribución, B. retama presentó valores del índice de vulnerabilidad menores a Vachellia aroma (Gillies ex Hook. \& Arn.) Seigler \& Ebinger $(5,8)$, Parkinsonia praecox (2), Geoffroea decorticans $(1,2)$; y mayores a Jodina rhombifolia (Hook. \& Arn.) Reissek $(0,2)$ y Maytenus viscifolia Griseb. $(0,4)$ (Giménez \& Moglia, 2017). 


\section{CONCLUSIONES}

Bulnesia retama es un arbusto que posee un leño xeromórfico muy denso y con características relacionadas con la resistencia a condiciones de aridez de los ambientes donde se distribuye. Presenta la combinación de rasgos anatómicos como vasos numerosos, pequeños y principalmente solitarios, fibras con paredes gruesas y parénquima axial apotraqueal. El incremento del área de fibras registrado en detrimento del área de parénquima y vasos, a medida que se incrementa la aridez, podría ayudar a predecir con este tipo de análisis, si un individuo de $B$. retama se encuentra en un sitio sumamente xérico (aportando mayor seguridad a la conducción de agua), o mésico (similar abundancia de parénquima y fibras, y mayor presencia de vasos). Esto permite considerar a la especie como altamente plástica frente a las condiciones de aridez, lo que podría explicar la amplitud del área de distribución de $B$. retama dentro de las Provincias Fitogeográficas del Monte y del Chaco Árido.

\section{AGRADECIMIENTOS}

A Fidel Roig, Eduardo Barrio y Martín Hadad por permitir y facilitar el análisis de los preparados histológicos en el Laboratorio de Anatomía Vegetal del IANIGLA. Este trabajo pudo realizarse gracias al aporte económico de los proyectos CICITCA-UNSJ (21E/891) e IDEA-SECITI 2014-0032, dirigidos por E. P.

\section{BIBLIOGRAFÍA}

Abraham, E.; H. F. Del Valle; F. Roig; L. Torres; J. O. Ares; F. Coronato \& R. Godagnone. 2009. Overview of the geography of the Monte Desert biome (Argentina). Journal of Arid Environments 73: 144-153. DOI: https://doi. org/10.1016/j.jaridenv.2008.09.028

Andreoni, D. F. 2014. Plantas leñosas y estrategias humanas en el sur de Mendoza: una aproximación arqueobotánica. Tesis Doctoral, Universidad Nacional de La Plata.

Baas, P.; F. W. Ewers; S. D. Davis \& E. A. Wheeler. 2004. Evolution of xylem physiology, en The evolution of plant physiology. Academic Press. DOI: https://doi.org/10.1016/ B978-012339552-8/50016-0
Barañao, J. J.; E. A. Peñón; E. Craig; E. Cucciufo \& P. De Falco. 2008. Manual para la identificación de maderas con aumentos de hasta 10x. Universidad Nacional De Luján, Departamento de Tecnología Producción Vegetal IV Dasonomía.

Bertonatti, C. 1997. Nuestro libro rojo. Vida Silvestre $N^{\circ} 56$ : 21-22, FVSA. Buenos Aires.

Böcher, T. W. \& O. B. Lyshede. 1968. Anatomical Studies in Xerophytic Apophyllous Plants. I. Montea aphylla, Bulnesia retama and Bredemeyera colletioides. Det Kongelige Danske Videnskabernes. Selskab Biologiske Skrifter 16(3): 1-44.

Burgerstein, A. 1912. Anatomische Untersuchungen argentinischer Hölzer des kk naturhistorischen Hofmuseums in Wien. Annalen des Naturhistorischen Museums in Wien 26(1/2): 1-36.

Cabrera, A. L. 1994. Regiones Fitogeográficas Argentinas. $1^{\circ}$ reimpresión, revisión a cargo de W. F. Kluger. Enciclopedia Argentina de Agricultura y Jardinería. Tomo 2, fasc. 1. Buenos Aires: Acme. (Publ. orig. 1976).

Carlquist, S. 1977. Ecological factors in wood evolution: a floristic approach. American Journal of Botany 64: 887-896. DOI: https://doi.org/10.1002/j.1537-2197.1977.tb11932.x

Carlquist, S. 1988. Comparative wood anatomy. Systematic, ecological and evolutionary aspect of Dicotyledons wood. Springer, Berlin, Heidelberg, New York. DOI: https://doi. org/10.1007/978-3-662-21714-6

CGIAR-CSI - Consortium for Spatial Information. 2021. Global aridity and PET database. https://cgiarcsi.community/data/ global-aridity-and-pet-database/

Chave, J. 2005 Measuring wood density for tropical forest trees. A field manual for the CTFS sites. Wood density measurement protocol. Lab. Evolution et Diversité Biologique. Université Paul Sabatier.

Chave, J.; D. Coomes; S. Jansen; S. L. Lewis; N. Swenson \& A. E. Zanne. 2009. Towards a worldwide wood economics spectrum. Ecology Letters 12: 351-366. DOI: https://doi. org/10.1111/j.1461-0248.2009.01285.x

Choat, B.; S. Jansen; T. J. Brodribb; H. Cochard; S. Delzon; R. Bhaskar \& A. E. Zanne. 2012. Global convergence in the vulnerability of forests to drought. Nature 491: 752-755. DOI: https://doi.org/10.1038/nature11688

Comas, C. I. \& J. H. Hunziker. 1996. Isozyme variation in Bulnesia retama, B. schickendantzii and B. foliosa (Zygophyllaceae). Plant Systematics and Evolution 199: 193-202. DOI: https://doi.org/10.1007/BF00984904

Cozzo, D. 1948 Anatomía del leño secundario de las especies argentinas de la tribu Zygophylleae (Zigofilaceas). Revista del Instituto Nacional de Investigación de las Ciencias Naturales y Museo Argentino de Ciencias Naturales Bernardino Rivadavia 1: 57-85. 


\section{E. A. MELIÁN ET AL. Variación de rasgos del xilema secundario de Bulnesia retama}

Crisci, J. V.; J. H. Hunziker; R. A. Palacios \& C. A. Naranjo. 1979. A numerical-taxonomic study of the genus Bulnesia (Zygophyllaceae): cluster analysis, ordination and simulation of evolutionary trees. American Journal of Botany 66: 133-140. DOI: https://doi.org/10.1002/j.1537-2197.1979. tb06205. $\mathrm{x}$

Dalmasso, A. D. \& J. A. Llera. 1996. Contenido de cera en relación al diámetro de ramas de Bulnesia retama en Ampacama, Caucete, San Juan. Multequina 5: 43-48.

Dalmasso, A. D.; J. Márquez; J. Carnino; J. Scaglia; M. Hadad; J. P. Cáceres; R. Brizuela \& A. Gómez. 2014. Especies apropiadas de arbolado para la provincia de San Juan. 1a ed.-San Juan: Universidad Nacional de San Juan. ISBN 978950-605-781-7.

D'ambrogio de Argüeso, A. 1986. Manual de técnicas en histología vegetal. Editorial Hemisferio Sur S.A.

Davis, S. D.; J. S. Sperry \& U. G. Hacke. 1999. The relationship between xylem conduit diameter and cavitation caused by freezing. American Journal of Botany 86: 1367-1372.

Debandi, G.; B. Rossi; J. Araníbar; J. A. Ambrosetti \& I. E. Peralta. 2002. Breeding system of Bulnesia retama (Gillies ex Hook \& Arn.) Gris. (Zygophyllaceae) in the Central Monte Desert (Mendoza, Argentina). Journal of Arid Environments 51: 141-152. DOI: https://doi.org/10.1006/ jare.2001.0924

Delignette-Muller, M. L. \& C. Dutang. 2015. fitdistrplus: An $\mathrm{R}$ package for fitting distributions. Journal of Statistical Software 64: 1-34. DOI: https://doi.org/10.18637/jss.v064. i04

García-Cervigón, A. I.; J. M. Olano; G. von Arx \& A. Fajardo. 2018. Xylem adjusts to maintain efficiency across a steep precipitation gradient in two coexisting generalist species. Annals of Botany 122: 461-472. DOI: https://doi. org/10.1093/aob/mcy088

Gatica, M. G. 2015. Cambios en la estructura de la vegetación a lo largo de un gradiente de lluvias en una región árida del centro-oeste de Argentina: consecuencias sobre la dinámica del carbono. Tesis Doctoral, Probiol, Universidad Nacional de Cuyo, Mendoza, Argentina.

Gatica, M. G.; J. N. Araníbar \& E. Pucheta. 2017. Environmental and species specific controls on $\delta 13 \mathrm{C}$ and $\delta 15 \mathrm{~N}$ in dominant woody plants from central western Argentinian drylands. Austral Ecology 42: 533-543. DOI: https://doi.org/10.1111/ aec. 12473

Giménez, A. M.; P. Hernández; R. Gerez \& C. Spagarino. 2007. Anatomía de leño y anillos de crecimiento de Palo Santo (Bulnesia sarmientoi Lorenz ex. Griseb Zygophyllaceae). Quebracho-Revista de Ciencias Forestales 14: 23-35.
Giménez, A. M. \& J. G. Moglia. 2003. Árboles del Chaco Argentino: guía para el reconocimiento dendrológico. Facultad de Ciencias Forestales. Universidad Nacional de Santiago del Estero. https://fcf.unse.edu.ar/index.php/ portfolio/arboles-del-chaco-argentino/\#

Giménez, A. M. \& J. G. Moglia. 2017. Los bosques actuales del Chaco semiárido argentino: ecoanatomía y biodiversidad: una mirada propositiva. Facultad de Ciencias Forestales, Universidad Nacional de Santiago del Estero. ISBN 978987-4078-12-4. https://fcf.unse.edu.ar/index.php/portfolio/ ecoanatomia-y-biodiversidad/?mode=grid

Godoy-Bürki, A. C.; J. M., Acosta \& L. Aagesen. 2018. Phylogenetic relationships within the New World subfamily Larreoideae (Zygophyllaceae) confirm polyphyly of the disjunct genus Bulnesia. Systematics and Biodiversity 16: 453-468. DOI: https://doi.org/10.1080/14772000.2018.14 51406

Hacke, U. G. \& J. S. Sperry. 2001. Functional and ecological xylem anatomy. Perspectives in Plant Ecology, Evolution and Systematics 4/2: 97-115. DOI: https://doi.org/10.1078/14338319-00017

Hacke, U. G.; R. Spicer; S. G. Schreiber \& L. Plavcov. 2017. An ecophysiological and developmental perspective on variation in vessel diameter. Plant, Cell \& Environment 40: 831-845. DOI: https://doi.org/10.1002/joc.1276

Hajek, P.; C. Leuschner; D. Hertel; S. Delzon \& B. Schuldt. 2014. Trade-offs between xylem hydraulic properties, wood anatomy and yield in Populus. Tree physiology 34: 744-756. DOI: https://doi.org/10.1093/treephys/tpu048

Hijmans, R. J.; S. E. Cameron; J. L. Parra; P. G. Jones \& A. Jarvis. 2005. Very high resolution interpolated climate surfaces for global land areas. International Journal of Climatology: 25: 1965-1978. DOI: https://doi.org/10.1002/ joc. 1276

Hunziker, J. H. 1994. Zygophyllaceae. Flora Fanerogámica Argentina 95: 1-20.

IAWA COMMITTEE. 1989. IAWA list of microscopic features for hardwood identification. IAWA Bulletin. 10: 219-332.

Jacobsen, A. L.; F. W. Ewers; R. B. Pratt; W. A. Paddock \& S. D. Davis. 2005. Do xylem fibers affect vessel cavitation resistance? Plant Physiology 139(1): 546-556. DOI: https:// doi.org/10.1104/pp.104.058404

Klein, T.; M. J. Zeppel; W. R. Anderegg; J. Bloemen; M. G. De Kauwe; P. Hudson \& A. Nardini. 2018. Xylem embolism refilling and resilience against drought-induced mortality in woody plants: processes and trade-offs. Ecological Research 33(5): 839-855. DOI: https://doi.org/10.1007/ s11284-018-1588-y 
Kiorapostolou, N.; L. Da Sois; F. Petruzzellis; T. Savi; P. Trifilò; A. Nardini \& G. Petit. 2019. Vulnerability to xylem embolism correlates to wood parenchyma fraction in angiosperms but not in gymnosperms. Tree physiology 39(10): 1675-1684. DOI: https://doi.org/10.1093/treephys/ tpz068

Kozlowski, T. T. 1992. Carbohydrate sources and sinks in woody plants. The Botanical Review 58(2): 107-222. DOI: https://doi.org/10.1007/BF02858600

Lindorf, H. 1994. Eco-anatomical wood features of species from a very dry tropical forest. International Association of Wood Anatomists Journal 15(4): 361-376. DOI: https://doi. org/10.1163/22941932-90001370

Maherali, H.; W. T. Pockman \& R. B. Jackson. 2004. Adaptive variation in the vulnerability of woody plants to xylem cavitation. Ecology 85(8): 2184-2199. DOI: https://doi. org/10.1890/02-0538

Martínez-Cabrera, H. I.; C. S. Jones; S. Espino \& H. J. Schenk. 2009. Wood anatomy and wood density in shrubs: Responses to varying aridity along transcontinental transects. American Journal of Botany 96(8): 1388-1398. DOI: https://doi. org/10.3732/ajb.0800237

Medeiros, J. S., \& W. T. Pockman. 2014. Freezing regime and trade-offs with water transport efficiency generate variation in xylem structure across diploid populations of Larrea sp. (Zygophyllaceae). American Journal of Botany 101(4): 598-607.

Moglia, J. G.; S. Bravo; A. M. Giménez \& C. López. 2009. ¿Son los caracteres estructurales de la madera de Aspidosperma quebracho-blanco Schelkt causantes de su inestabilidad?. Quebracho-Revista de Ciencias Forestales 17(1-2): 58-63. https://fcf.unse.edu.ar/archivos/quebracho/ v17a07.pdf

Morello, J. 1958. La Provincia Fitogeográfica del Monte. Opera Lilloana 2: 1-155.

Morris, H.; L. Plavcová; P. Cvecko; E. Fichtler; M. A. Gillingham; H. I. Martínez Cabrera \& S. Jansen. 2016. A global analysis of parenchyma tissue fractions in secondary xylem of seed plants. New Phytologist 209(4): 1553-1565. DOI: https://doi.org/10.1111/nph.13737

Morris, H.; M. A. Gillingham; L. Plavcová; S. M. Gleason; M. E. Olson; D. A. Coomes \& E. A. Wheeler. 2018. Vessel diameter is related to amount and spatial arrangement of axial parenchyma in woody angiosperms. Plant, Cell \& Environment 41(1): 245-260. DOI: https://doi.org/10.1111/ pce. 13091

Palacios, R. A. \& J. H. Hunziker. 1984. Revisión taxonómica del género Bulnesia (Zygophyllaceae). Darwiniana 25: 299320 .
Pérez-Harguindeguy, N.; S. Díaz; E. Garnier; S. Lavorel; H. Poorter; P. Jaureguiberry \& C. Urcelay. 2013. New handbook for standardized measurement of plant functional traits worldwide. Australian Journal of Botany 61: 167-234. DOI: https://doi.org/10.1071/BT12225

Pratt, R. B. \& A. L. Jacobsen. 2017. Conflicting demands on angiosperm xylem: tradeoffs among storage, transport and biomechanics. Plant, Cell \& Environment 40(6): 897-913. DOI: https://doi.org/10.1111/pce.12862

Porter, D. M. 1974. Disjunct distributions in the New World Zygophyllaceae. Taxon 23: 339-346. DOI: https://doi. org/10.2307/1218714

QGIS Development Team. 2020. QGIS Geographic Information System. Open Source Geospatial Foundation Project. Retrieved from http://qgis.osgeo.org/

R CORE TEAM. 2018. R: A language and environment for statistical computing. R Foundation for Statistical Computing, Vienna, Austria. https://www.R-project.org/

Rasband, W.S. ImageJ, U. S. National Institutes of Health, Bethesda, Maryland, USA, https://imagej.nih.gov/ij/, 1997-2018.

Record, S. J. 1921. Lignum-vitae: A Study of the Woods of the Zygophyllaceae with Reference to the True Lignum-vitae of Commerce--its Sources, Properties, Uses, and Substitutes (6). Yale University, School of Forestry. DOI: https://doi. org/10.5962/bhl.title.43550

Ribas-Fernández, Y. 2016. Importancia de la disponibilidad de agua y de los parches de arbustos como reguladores del destino de semillas y el establecimiento de plántulas de Bulnesia retama (Zygophyllaceae) a lo largo de un gradiente de precipitaciones en el Desierto del Monte. Tesis Doctoral. Universidad Nacional de Córdoba.

Ribas-Fernández, Y.; L. Quevedo-Robledo \& E. Pucheta. 2009. Pre-and post-dispersal seed loss and soil seed dynamics of the dominant Bulnesia retama (Zygophyllaceae) shrub in a sandy Monte desert of western Argentina. Journal of Arid Environments 73: 14-21. DOI: https://doi.org/10.1016/j.jaridenv.2007.12.001

Rodríguez Rivera, M.; E. Fernández; L. Sosa; V. Villareal \& M. Reale.2007. Efecto del estrés hídrico a distintas temperaturas sobre la germinación de semillas de Bulnesia retama. PHYTON 76: 5-17.

Rosas, T.; M. Mencuccini; J. Barba; H. Cochard; S. Saura-Mas \& J. Martínez Vilalta. 2019. Adjustments and coordination of hydraulic, leaf and stem traits along a water availability gradient. New Phytologist 223: 632-646. DOI: https://doi. org/10.1111/nph.15684

Rosell, J. A.; M. E. Olson \& T. Anfodillo. 2017. Scaling of xylem vessel diameter with plant size: causes, predictions, and outstanding questions. Current Forestry Reports 3: 4659. DOI: https://doi.org/10.1007/s40725-017-0049-0 


\section{E. A. MELIÁN ET AL. Variación de rasgos del xilema secundario de Bulnesia retama}

Ruzin, S. E. 1999. Plant microtechnique and microscopy. Oxford University Press. New York.

Sarmiento, C.; S. Patiño; C. E. T. Paine; J. Beauchene; A. Thibaut \& C. Baraloto. 2011. Within-individual variation of trunk and branch xylem density in tropical trees. American Journal of Botany 98:140-149. DOI: https://doi. org/10.3732/ajb.1000034

Schenk, H. J.; S. Espino; C. M. Goedhart; M. Nordenstahl; H. I. M. Cabrera \& C. S. Jones. 2008. Hydraulic integration and shrub growth form linked across continental aridity gradients. Proceedings of the National Academy of Sciences 105: 1124811253. DOI: https://doi.org/10.1073/pnas.0804294105

Smith, D. M. 1967. Microscopic methods for determining cross-sectional cell dimensions. US Forest Service Research Paper FPL 79.

Sosa, L. \& E. Fernández. 1998. Resultados preliminares en estudios sobre germinación y supervivencia al trasplante de Bulnesia retama "retamo" en la provincia de San Luis. Actas XXVI Jornadas Argentinas de Botánica (Río Cuarto, Córdoba) 174:367.

Sperry, J. S. 2003. Evolution of water transport and xylem structure. International Journal of Plant Sciences 164: S115-S127. DOI: https://doi.org/10.1086/368398

Sperry, J. S.; F. C. Meinzer \& K. A. McCulloh. 2008. Safety and efficiency conflicts in hydraulic architecture: scaling from tissues to trees. Plant, Cell and Environment 31: 632-645. DOI: https://doi.org/10.1111/j.1365-3040.2007.01765.x

Sperry, J. S. \& D. M. Love. 2015. What plant hydraulics can tell us about responses to climate-change droughts. New Phytologist 207: 14-27. DOI: https://doi.org/10.1111/nph.13354
Tyree, M. T. \& J. S. Sperry. 1989. Vulnerability of xylem to cavitation and embolism. Annual Review of Plant Biology 40: 19-36. DOI: https://doi.org/10.1146/annurev. pp.40.060189.000315

Tyree, M. T. \& M. H. Zimmermann. 2013. Xylem structure and the ascent of sap. Springer Science \& Business Media. DOI: https://doi.org/10.1007/978-3-662-22627-8

Tortorelli, L.A. 1956. Maderas y bosques argentinos. Editorial Acme, Buenos Aires.

Villar-Salvador, P.; P. Castro-Diez; C. Pérez-Rontomé \& G. Montserrat-Martí.1997. Stem xylem features in three Quercus (Fagaceae) species along a climatic gradient in NE Spain. Trees 12: 90-96. DOI: https://doi.org/10.1007/ PL00009701

Violle, C.; M. L. Navas; D. Vile; E. Kazakou; C. Fortunel; I. Hummel \& E. Garnier. 2007. Let the concept of trait be functional! Oikos 116: 882-892. DOI: https://doi. org/10.1111/j.0030-1299.2007.15559.x

Wheeler, E. A. \& P. Baas. 1998. Wood identification-a review. IAWA Journal 19: 241-264. DOI: https://doi. org/10.1163/22941932-90001528

Ziemińska , K.; D. W. Butler; S. M. Gleason; I. J. Wright \& M. Westoby. 2013. Fibre wall and lumen fractions drive wood density variation across 24 Australian angiosperms. $A o B$ Plants 5. DOI: https://doi.org/10.1093/aobpla/plt046

Ziemińska, K.; M. Westoby \& I. J. Wright. 2015. Broad anatomical variation within a narrow wood density range-A study of twig wood across 69 Australian angiosperms. PLoS One 10(4). DOI: https://doi.org/10.1371/journal. pone.0124892 